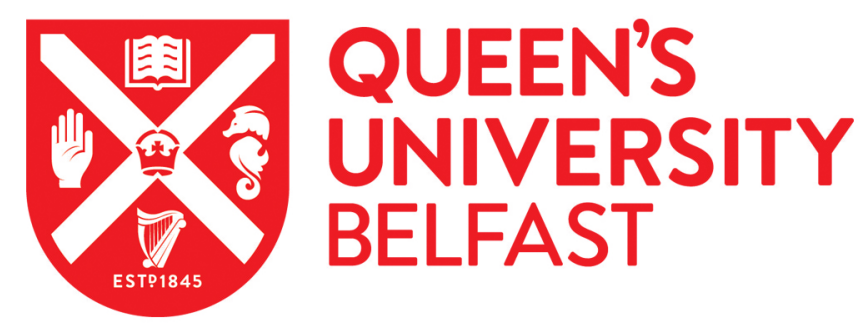

\title{
Insights into the Mechanism of Action of the Two-Peptide Lantibiotic Lacticin 3147
}

\author{
Bakhtiary, A., Cochrane, S. A., Mercier, P., McKay, R. T., Miskolzie, M., Sit, C. S., \& Vederas, J. C. (2017). \\ Insights into the Mechanism of Action of the Two-Peptide Lantibiotic Lacticin 3147. Journal of the American \\ Chemical Society, 139(49), 17803-17810. https://doi.org/10.1021/jacs.7b04728
}

\section{Published in:}

Journal of the American Chemical Society

\section{Document Version:}

Peer reviewed version

\section{Queen's University Belfast - Research Portal:}

Link to publication record in Queen's University Belfast Research Portal

\section{Publisher rights}

Copyright 2017 ACS. This work is made available online in accordance with the publisher's policies. Please refer to any applicable terms of use of the publisher.

\section{General rights}

Copyright for the publications made accessible via the Queen's University Belfast Research Portal is retained by the author(s) and / or other copyright owners and it is a condition of accessing these publications that users recognise and abide by the legal requirements associated with these rights.

Take down policy

The Research Portal is Queen's institutional repository that provides access to Queen's research output. Every effort has been made to ensure that content in the Research Portal does not infringe any person's rights, or applicable UK laws. If you discover content in the Research Portal that you believe breaches copyright or violates any law, please contact openaccess@qub.ac.uk. 


\title{
Insights into the Mechanism of Action of the Two-Peptide Lantibiotic Lacticin 3147
}

\author{
Alireza Bakhtiary, ${ }^{\dagger}$ Stephen A. Cochrane,${ }^{\S}$ Pascal Mercier,${ }^{\dagger}$ Ryan T. McKay,${ }^{\dagger}$ Mark Miskolzie,${ }^{\dagger}$ Clarissa \\ S. Sit ${ }^{\text {tl }}$ and John C. Vederas ${ }^{\dagger *}$ \\ 'Department of Chemistry, University of Alberta, Edmonton, AB, Canada, T6G 2G2; ${ }^{\S}$ School of Chemistry and Chemical \\ Engineering, Queens University Belfast, UK, BT9 5AG; *National High Field NMR Centre, University of Alberta, \\ Edmonton, AB, Canada, T6G 2E1; "Department of Chemistry, Saint Mary's University, Halifax, NS, Canada, B3H 3C3
}

Supporting Information Placeholder

\begin{abstract}
Lacticin 3147 is a two peptide lantibiotc (LtnA1 and LtnA2) that displays nanomolar activity against many Gram-positive bacteria. Lacticin 3147 may exert its antimicrobial effect by several mechanisms. Isothermal titration calorimetry experiments show that only LtnA1 binds to the peptidoglycan precursor lipid II, which could inhibit peptidoglycan biosynthesis. An experimentally supported model of the resulting complex that suggests the key binding partners are the C-terminus of LtnA1 and pyrophosphate of lipid II. A combination of in vivo and in vitro assays indicates that LtnA1 and LtnA2 can induce rapid membrane lysis without the need for lipid II binding. However, the presence of lipid II substantially increases the activity of lacticin 3147. Furthermore, studies with synthetic LtnA2 analogues containing either desmethyl- or oxalanthionine rings confirm that the precise geometry of these rings is essential for this synergistic activity.
\end{abstract}

\section{INTRODUCTION}

The continued emergence of antibiotic-resistant bacteria necessitates the discovery of novel antimicrobial compounds. ${ }^{1}$ In recent years, antimicrobial peptides (AMPs) have been the vanguard of new antimicrobial agents. ${ }^{2}$ Bacteriocins are a class of AMPs produced by bacteria that often display strong activity against pathogenic bacterial strains. ${ }^{3}$ The lantibiotics are a subclass of bacteriocins defined by their sulfur-containing rings, which result from the 1,4-conjugate addition of cysteine to dehydroalanine (lanthionine) or dehydrobutyrine (methyllanthionine) during their biosynthesis. ${ }^{4}$ Understanding the mechanism of action of antimicrobial compounds is important, as it can enable the rational design of more active and/or synthetically more accessible analogues. ${ }^{5}$ For lantibiotics, this can be achieved by chemical synthesis or genetic engineering. ${ }^{4-6}$ A significant number of known AMPs bind to the peptidoglycan precursor lipid II. Peptidoglycan is structurally essential and unique to bacteria, and many antibiotics target this pathway. ${ }^{7}$ Lipid II binding AMPs can exert their antimicrobial effect through a variety of mechanisms. This includes inhibition of peptidoglycan biosynthesis (vancomycin, plectasin and teixobactin), ${ }^{8,9,2 \mathrm{a}}$ disruption of the proton-motive-force (tridecaptin $\left.\mathrm{A}_{1}\right)^{10}$ and membrane lysis/pore formation (lantibiotics). ${ }^{11}$

Lantibiotics are grouped into Type-A and -B peptides, based on their functional and structural features. ${ }^{12}$ Type-A lantibiotics are elongated, with similar arrangements of their thioether bridges. This group is subdivided into Type-A(I) and Type-A(II). Type-A(I) lantibiotics contain several charged residues and have a net negative or slightly positive charge whereas Type-A(II) lantibiotics are highly negatively charged. Type-B lantibiotics are typically shorter than Type-A and adopt a globular structure. Although these types differ considerably, examples from all types have been shown to bind lipid II.

Nisin (1) is a Type-A(I) lantibiotic routinely used in the food industry to protect against pathogenic Gram-positive bacteria (Figure 1). ${ }^{13}$ Nisin exerts its antimicrobial effect by pore formation on bacterial membranes, which is enhanced by the presence of lipid II, ${ }^{14}$ and binds to lipid II through its N-terminal lanthionine rings, forming a "pyrophosphate cage". ${ }^{4 \mathrm{~d}}$ Mersacidin (2) is a Type-B lantibiotic that also binds to lipid II. ${ }^{15}$ 2D-NMR experiments have shown that when lipid II is added to mersacidin, it undergoes several chemical shift perturbations in its C-terminus. ${ }^{15 \mathrm{~b}}$ There are also two component lantibiotic systems wherein one peptide binds to lipid II. These two component lantibiotics work synergistically to kill bacteria and include the haloduracins $^{16}(\mathrm{Hal} \alpha(3)$ and $\beta(4))$ and lacticin 3147 (LtnA1 (5) and A2 (6)). ${ }^{17} \mathrm{Hal} \alpha$ and LtnA1 are Type-Blike lantibiotics with lanthionine bridging patterns $(\mathrm{B}, \mathrm{C}$ and $\mathrm{D}$ rings) very similar to mersacidin. Mutations in these areas have been shown to reduce antimicrobial activity. ${ }^{18,19} \mathrm{Hal} \beta$ and LtnA2 are both Type-A(I)-like lantibiotics with overall charges of +1 . The similarities between mersacidin, Hal $\alpha$ and LtnA1 are suggestive of a conserved lipid II binding motif. ${ }^{15,16}$ 

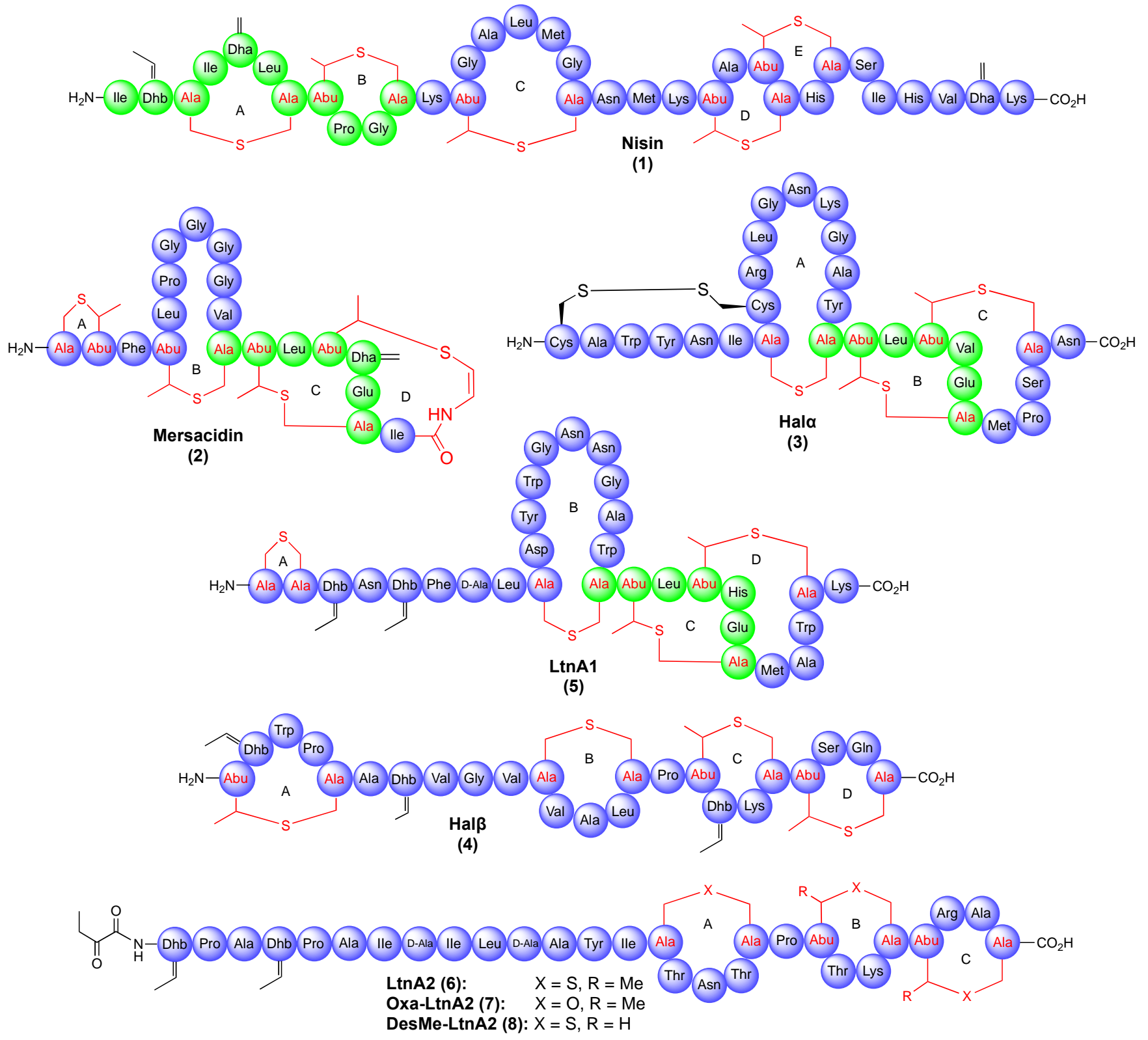

Figure 1. Bead structures of some lipid II-binding lantibiotics. Residues known or suspected to be involved in lipid II binding are light green. Lanthionine, methyl lanthionine and amino-vinyl-cysteine bridges are red. Type-A $(\mathrm{I})=$ nisin $(\mathbf{1})$; and Type-B = mersacidin (2). Haloduracin and Lacticin 3147 are two-component lantibiotics, in which Hal $\alpha$ (3) and LtnA1 (5) are Type-B-like and bind to lipid II. Hal $\beta$ (4) and LtnA2 (6) are type-A(I) like but do not bind to lipid II. Synthetic oxa-LtnA2 (7) and desMe-LtnA2 (8) are also shown.

Lacticin 3147 is produced by Lactococcus lactis subspecies lactis DPC3147 and has broad-spectrum activity against Gram-positive bacteria. ${ }^{17}$ Previous mechanistic studies revealed that LtnA1 binds to lipid II on the surface of the cell membrane. ${ }^{20}$ It has been proposed that this complex then recruits $\operatorname{Ltn} \mathrm{A} 2$ and the resulting ternary complex inhibits cell wall synthesis and causes pore formation. Previous activity assays have shown that LtnA1 and LtnA2 operate in a 1:1 stoichiometry. ${ }^{17 \mathrm{~d}}$ Our group has previously synthesized oxa- and desmethyl-lanthionine analogues of LtnA2 (oxaLtnA2 (7) and desMe-LtnA2 (8)), wherein lanthionine sulfurs are replaced with oxygen or methyls are missing from methyllanthionine residues, respectively. ${ }^{21,22} \mathrm{We}$ also completed the first total syntheses of LtnA1 and LtnA2. ${ }^{23}$ Mechanistic studies on other lipid II binding peptides inspired us to study the mechanism of action of lacticin 3147 and the structure of the LtnA1 interaction with lipid II. ${ }^{10}$

\section{RESULTS AND DISCUSSION Isothermal Titration Calorimetry}

Isothermal titration calorimetry (ITC) is commonly used to assess the lipid II binding properties of antimicrobial peptides. ${ }^{24,25,10}$ We used ITC to study the interactions between natural and synthetic analogues of lacticin 3147 

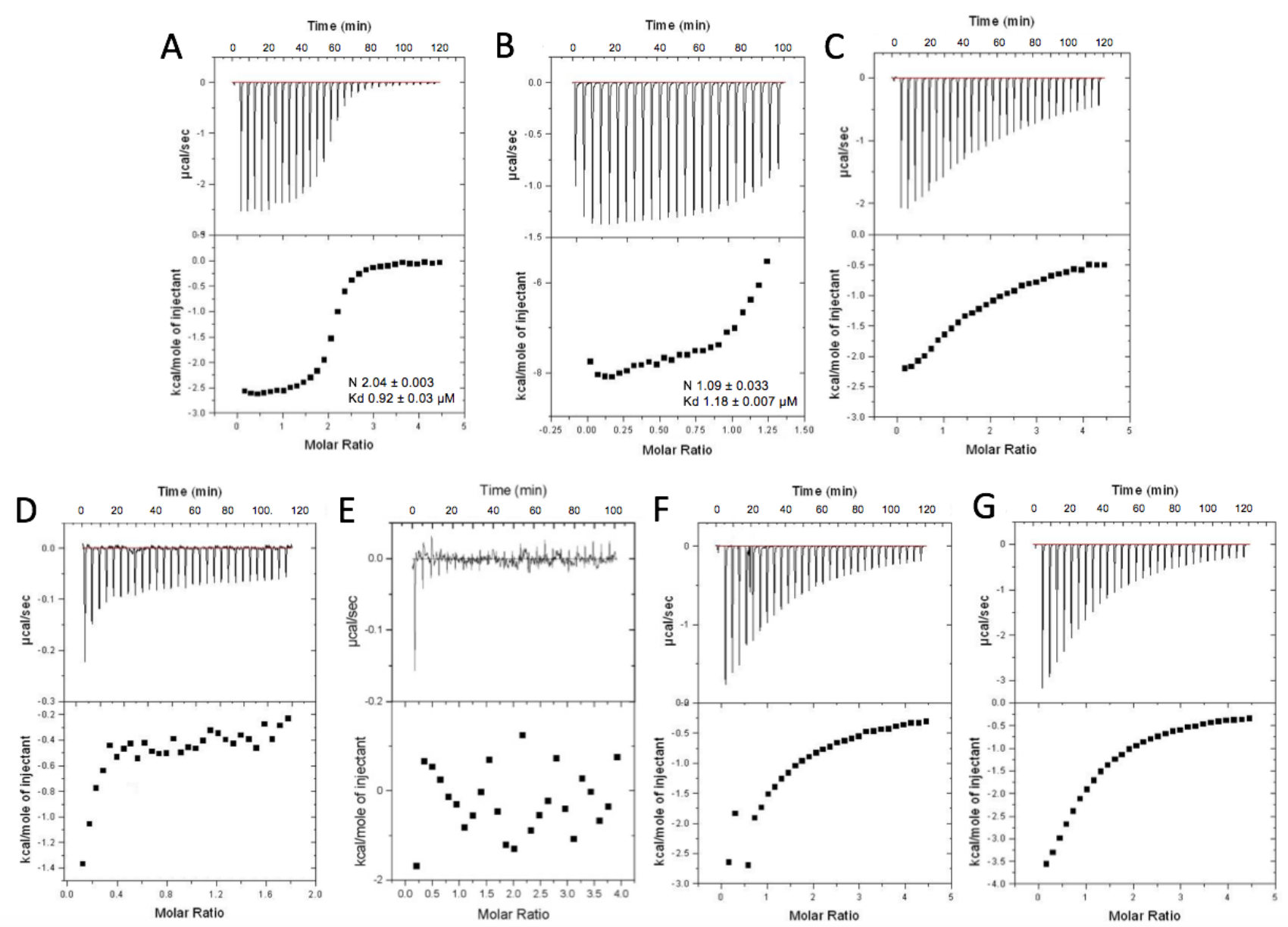

Figure 2. ITC binding experiments with natural and synthetic lacticin 3147 analogues and phospholipid LUVs containing 1 mol\% Gram-positive lipid II. (A) Lipid II added to LtnA1. (B) LtnA1 added to LtnA2. (C) Lipid II added to 1:1 LtnA1:LtnA2. (D) LtnA1 added to desMe-LtnA2. (E) LtnA1 added to oxa-LtnA2. (F) Lipid II added to 1:1 LtnA1:desMe-LtnA2. (G) Lipid II added to 1:1 LtnA1:oxa-LtnA2. Binding enthalpy $(\mathrm{Kd})$ and number of binding sites $(\mathrm{N})$ are shown.

peptides, as well as synergistic mixtures, and Grampositive lipid II. Gram-positive lipid II often contains lysine in its pentapeptide, whereas Gram-negative lipid II usually contains diaminopimelic acid. To better mimic a Gram-positive bacterial membrane, 1 mol\% lipid II (containing a lysine residue) was incorporated into large unilamellar vesicles (LUVs) for the ITC experiments. In these LUVs, lipid II is symmetrically distributed between the inner and outer leaflets, and cannot translocate the artificial membrane without a flippase. Therefore, the actual concentration of lipid II present on the outer leaflet is $0.5 \mathrm{~mol} \% .{ }^{26} \mathrm{LtnA} 1$ binds to membrane-embedded lipid II in a $2: 1$ ratio with a binding affinity $\left(k_{\mathrm{D}}\right)$ of $0.9 \mu \mathrm{M}$, which is consistent with its minimum inhibitory concentration $(1 \mu \mathrm{M})$ (Figure $2 \mathrm{~A})$. However, none of the LtnA2 analogues bind to lipid II (Figure S1). Given the synergistic relationship between LtnA1 and LtnA2, the binding interaction between these peptides was also assessed by ITC. LtnA1 binds strongly to LtnA2 in a 1:1 ratio with a $k_{\mathrm{D}}$ of $1.2 \mu \mathrm{M}$ (Figure 2B). When lipid II LUVs were added to a $1: 1$ mixture of $\operatorname{LtnA} 1: \operatorname{LnA} 2$, the binding isotherm changed (Figure 2C). The resulting isotherm is characteristic of a heterotropic interaction, in which cooperative binding occurs between a macromolecule (LtnA1) and two different ligands (LtnA2 and lipid II). ${ }^{27}$ Therefore it is not accurate to measure the number of binding sites or binding affinity by this method as it only gives an average of the various possible binding states between LtnA1, LtnA2 and lipid II embedded in the LUVs. The binding of LtnA1 to synthetic LtnA2 analogues was also tested, and it was found that LtnA1 binds weakly to desMe-LtnA2 (Figure 2D) and does not bind to oxa-LtnA2 at all (Figure 2E). These measurements are consistent with our previous observations that desMe-LtnA2 has reduced synergistic activity with LtnA1 but oxa-LtnA2 is not synergistic with LtnA1. ${ }^{21,22}$ The binding of LtnA1 + des-LtnA2 to lipid II (Figure 2F) and LtnA1 + oxa-LtnA2 to lipid II (Figure $2 \mathrm{G})$ were then analyzed. The resulting curves are very similar to $\operatorname{Ltn} A 1+\operatorname{Ltn} A 2+$ lipid II (Figure 2C). In the case of oxa-LtnA2 this is surprising, given that it does not bind to LtnA1. It is possible that upon formation of the LtnA1:lipid II complex, oxa-LtnA2 is then able to bind to this structure. However, at this stage further experiments were required to determine how these binding studies relate to antimicrobial activity. 

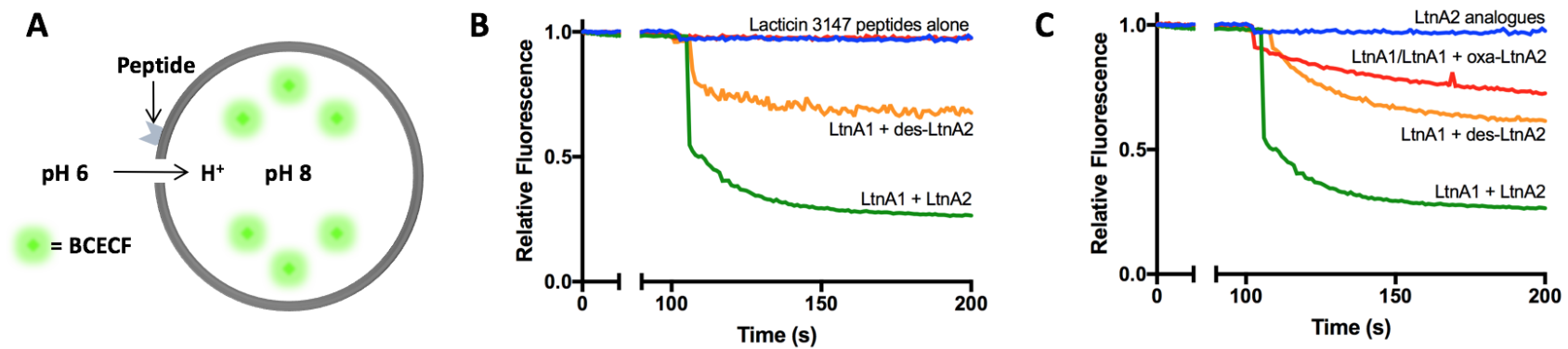

Figure 3. (A) Cartoon schematic of the assay used to assess membrane lysis of the lacticin 3147 analogues. (B) Relative fluorescence vs. time graph showing the effects of adding lacticin 3147 analogues to LUVs containing BCECF. Peptide concentrations are $5 \mu \mathrm{M}$. (C) Relative fluorescence vs. time graph showing the effects of adding lacticin 3147 analogues to LUVs doped with 1 mol\% lipid II and containing BCECF. Peptide concentrations are $100 \mathrm{nM}$.

\section{In vitro membrane lysis assays}

To link the ITC results to the effect on cell membranes, a series of pore-formation assays were performed using LUVs containing the $\mathrm{pH}$ sensitive dye 2',7'-bis-(2carboxyethyl)-5-carboxy-fluorescein (BCECF). ${ }^{28}$ In this assay, LUVs are constructed with an internal $\mathrm{pH}$ of 8 and then placed in buffer at $\mathrm{pH} 6$. If pore formation occurs, there is a decrease in intra-vesicle $\mathrm{pH}$, which is observed as a decrease in BCECF fluorescence (Figure 3A). ${ }^{10,28}$ To assess the effect of membrane bound lipid II on membrane lysis, LUVs with and without lipid II were constructed. In LUVs lacking lipid II, no membrane lysis was observed for any of the lacticin 3147 analogues at concentrations up to $5 \mu \mathrm{M}$ (Figure 3B). However, a 1:1 mixture of LtnA1:LtnA2 caused rapid membrane lysis. The synergistic mixture of LtnA1:desMe-LtnA2 caused a smaller degree of membrane lysis in vesicles lacking lipid II, however no effect was observed for LtnA1:oxa-LtnA2. These results are consistent with the ITC studies, which showed that oxa-LtnA2 does not bind to LtnA1. When lipid II was incorporated into the LUVs, the concentration of 1:1 LtnA1:LtnA2 or 1:1 LtnA1:desMe-LtnA2 required for membrane lysis dropped significantly to $100 \mathrm{nM}$ (Figure 3C). Consistent with the absence of lipid II binding found during the ITC studies, none of the LtnA2 analogues caused membrane lysis. In contrast, LtnA1, which was shown by ITC to bind to lipid II, causes moderate membrane lysis at $100 \mathrm{nM}$. These results show that although lacticin 3147 can cause membrane lysis without lipid II, the presence of lipid II significantly enhances activity. An anomaly observed during the ITC studies was the binding curve for 1:1 LtnA1:oxa-LtnA2 + lipid II. Even though oxa-LtnA2 does not bind to LtnA1, the binding curve was nearly identical to the synergistic mixture of the natural peptides + lipid II, suggesting a similar interaction. However, the degree of membrane lysis observed for 1:1 LtnA1:oxa-LtnA2 is identical to LtnA1 alone, showing that the major interaction resulting in the ITC binding curve is LtnA1 binding to lipid II. Having linked lipid II binding to membrane lysis, we next sought to relate this to antimicrobial activity.

\section{Time-kill assays}

Time-kill assays are often used to determine if an antibiotic is bacteriostatic or bactericidal. ${ }^{10}$ In these experiments, Lactococcus lactis subsp. cremoris HP cells were incubated in the presence of set concentrations of lacticin 3147 peptides $(1 \mu \mathrm{M}$ or $250 \mathrm{nM})$ for 8 hours. At certain time points, an aliquot of bacterial suspension was removed, spread on an agar plate and grown overnight. The number of colony forming units (CFUs) per $\mathrm{mL}$ were then counted and plotted over the course of the 8-hour experiment (Figure 4A and B). In a time-kill assay, bacteriostatic agents (e.g. chloramphenicol) are easily identified, as the number of CFUs will remain constant over the time-course of the experiment. Bactericidal agents kill cells, therefore the number of CFUs will decrease over time if cells are exposed to a bactericidal compound. Furthermore, membrane lysing agents cause rapid cell death, with the number of CFUs typically being reduced to 0 within $30 \mathrm{~min}$. At both $250 \mathrm{nM}$ and $1 \mu \mathrm{M}$, LtnA1 halted cell-growth but did not significantly reduce the number of CFUs over an 8-hour period. This shows that LtnA1 binding to lipid II exerts a bacteriostatic effect. In contrast, LtnA2 is bactericidal. At $250 \mathrm{nM}$ all cells are killed over the 8-hour time-course, however at 1 $\mu \mathrm{M}$ the time taken to kill all cells is reduced to $30 \mathrm{~min}$. This suggests that at higher concentrations, LtnA2 alone can kill Gram-positive bacteria by lysis of the cell membrane. In the in vitro membrane lysis assay, LtnA2 showed no lytic activity against LUVs with or without lipid II. Therefore, these artificial membranes either lack the lipid composition required for LtnA2 induced membrane lysis, or LtnA2 binds to a membrane embedded biomolecule that is not present in the LUVs. To confirm this, an in vivo membrane lysis assay was required (next page). The synergistic mixture of LtnA1 and LtnA2 kills all cells within $10 \mathrm{~min}$ at $250 \mathrm{nM}$ and 5 min at $1 \mu \mathrm{M}$, which is highly suggestive of rapid membrane lysis. These results corroborate that the synergistic mixture of LtnA1 and LtnA2 kills Grampositive bacteria by rapid membrane lysis. To confirm these findings in vivo, SYTOX Green membrane lysis assays were performed. 

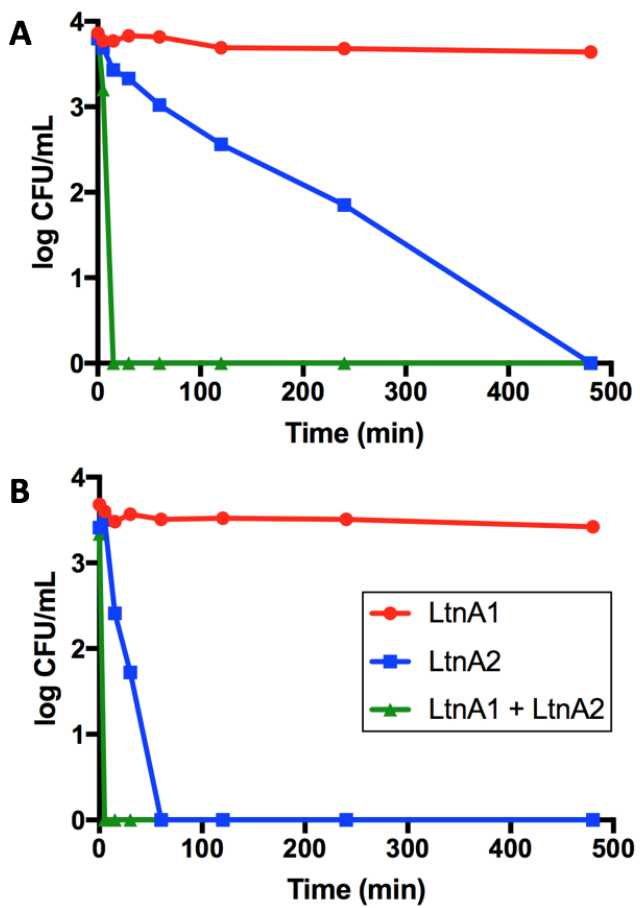

Figure 4. Time-kill assays. Lactococcus lactis subsp. cremoris HP cells treated with $250 \mathrm{nM}(\mathbf{A})$ or $1 \mu \mathrm{M}(\mathbf{B})$ of peptide and the number of viable cells determined at different time points.

\section{In vivo membrane lysis assays}

To assess the effect of lacticin 3147 peptides on wholecell membranes, in vivo membrane lysis assays were performed using SYTOX Green (Figure 5). SYTOX Green is a membrane-impermeable reagent that penetrates cells with compromised cell membranes, binding to nucleic acids and emitting a fluorescent signal. ${ }^{29}$ Lactococcus lactis subsp. cremoris HP cells were incubated with SYTOX Green and peptides were then added to a final concentration of $100 \mathrm{nM}$. Nisin (1) is known to cause rapid pore-formation at this concentration, and indeed showed rapid membrane lysis in the SYTOX Green assay. LtnA1 did not cause membrane lysis, which is consistent with the previous experiments in this study. The synergistic mixture of LtnA1 and LtnA2 caused immediate membrane lysis, although not to the same extent as nisin. Gratifyingly, LtnA2, which did not cause membrane lysis in the in vitro assay but showed bactericidal kinetics consistent with a lytic peptide, caused membrane lysis in this whole-cell experiment. This provides further evidence that either the artificial membranes lack the lipid content required for LtnA2 lysis, or they lack a membrane embedded biomolecule that LtnA2 binds to induce membrane lysis.

\section{Solution NMR derived model of LtnA1:lipid II complex}

Having identified LtnA1 as a lipid II binding peptide, we next sought to identify how it binds using NMR experiments. Natural lipid II contains a long undecaprenyl chain $\left(\mathrm{C}_{55}\right)$ that is not very amenable to NMR studies, as it has poor solubility and its multiple methylene units can

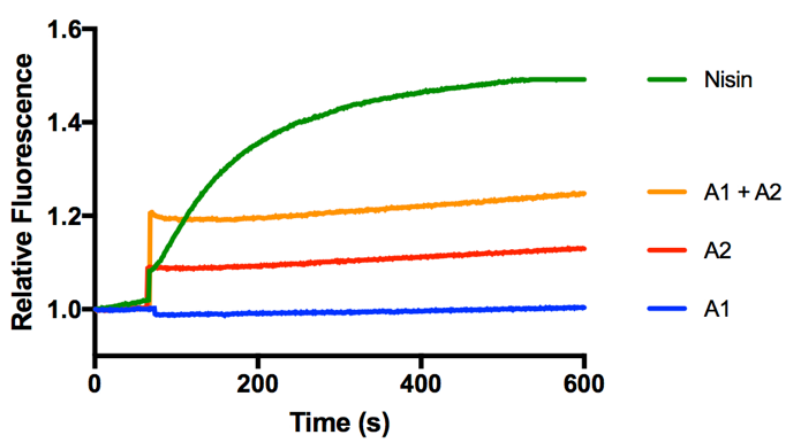

Figure 5. Lactococcus lactis subsp. cremoris HP cells pretreated with SYTOX green were exposed to antimicrobial peptides $(100 \mathrm{nM})$ and the extent of membrane lysis visualized as an increase in fluorescence.

important signals from the peptide. Therefore, a shorter chain version, $(E, E)$-farnesyl lipid II (9) was synthesized by adapting previously published methodology. ${ }^{10}$ Glycosyl acceptor $\mathbf{1 0}$ was prepared in 5 steps and glycosyl donor 11 in 4 steps from commercially available starting materials. ${ }^{10}$ A TMSOTf catalyzed glycosylation, followed by protecting group manipulation, phosphorylation of the MurNAc anomeric position and deprotection of the 2-(phenylsulfonyl)ethyl ester with DBU gave acid 12 (Scheme 1). Coupling to deprotected Boc-tetrapeptide 13 (Scheme S1) then produced disaccharidyl pentapeptide 14 in 54\% yield. Finally, deprotection of the dibenzyl phosphate moiety, followed by coupling to CDI activated $(E, E)$-farnesyl phosphate and HPLC purification yielded (E,E)-farnesyl lipid II (9) in $15 \%$ isolated yield.
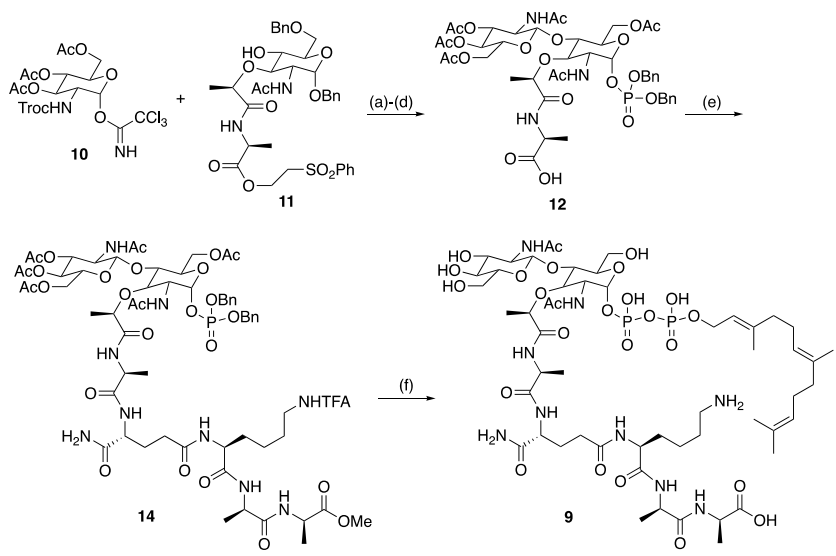

Scheme 1. Total synthesis of $(E, E)$-farnesyl lipid II (9). (a) TMSOTf, $4 \AA \mathrm{MS}, \mathrm{CH}_{2} \mathrm{Cl}_{2}, \mathrm{rt}, 18 \mathrm{~h}, 61 \%$. (b) (i) $\mathrm{ZnCl}_{2}$, $\mathrm{AcOH} / \mathrm{Ac}_{2} \mathrm{O}$, rt, 24 h; (ii) $\mathrm{Zn}, \mathrm{THF} / \mathrm{AcOH} / \mathrm{Ac}_{2} \mathrm{O}$, rt, 24 h, 63\% (two steps). (c) (i) $\mathrm{H}_{2}, \mathrm{Pd} / \mathrm{C}, \mathrm{MeOH}, \mathrm{rt}, 3 \mathrm{~h}$; (ii) ('Pr)${ }_{2} \mathrm{NP}(\mathrm{OBn})_{2}$, tetrazole, $\mathrm{CH}_{2} \mathrm{Cl}_{2}, \mathrm{rt}, 2 \mathrm{~h}$; (iii) $30 \% \mathrm{H}_{2} \mathrm{O}_{2}$ in $\mathrm{THF}$, $-78{ }^{\circ} \mathrm{C}$ to rt, $2 \mathrm{~h}, 84 \%$ (three steps). (d) $\mathrm{DBU}, \mathrm{CH}_{2} \mathrm{Cl}_{2}, \mathrm{rt}, 0.5$ h, quant. (e) Boc-Tetrapeptide 13, TFA $/ \mathrm{CH}_{2} \mathrm{Cl}_{2}, 2 \mathrm{~h}$; NHS, EDCI.HCl, DMF, rt, 18 h, 54\%. (f) (i) $\mathrm{H}_{2}, \mathrm{Pd} / \mathrm{C}, \mathrm{MeOH}, \mathrm{rt}, 2.5$ h, (ii) CDI activated $(E, E)$-farnesyl phosphate, DMF, rt, 4d, (iii) $\mathrm{NaOH}, \mathrm{H}_{2} \mathrm{O} /$ dioxane, $37^{\circ} \mathrm{C}, 2 \mathrm{~h}, 15 \%$ (three steps). 
Extensive solvent screening identified 1:1:1 $\mathrm{CD}_{3^{-}}$ $\mathrm{OH}: \mathrm{CD}_{3} \mathrm{CN}: \mathrm{H}_{2} \mathrm{O}$ as a suitable solvent for the NMR studies. LtnA1 and $(E, E)$-farnesyl lipid II were both characterized separately in this solvent system by ${ }^{1} \mathrm{H}-$ NMR, ${ }^{1} \mathrm{H}^{-1} \mathrm{H}$ COSY, ${ }^{1} \mathrm{H}-{ }^{-1} \mathrm{H}$ TOCSY, ${ }^{1} \mathrm{H}^{-1} \mathrm{H}$ NOESY and ${ }^{31} \mathrm{P}-\mathrm{NMR}$ (Tables S1-S2). The chemical shifts were then assigned in a 1:1 mixture of LtnA1 and $(E, E)$-farnesyl lipid II (Tables S3-S4). A significant chemical shift perturbation $(\sim 1.0 \mathrm{ppm})$ was observed in the pyrophosphate signals of lipid II when LtnA1 was added (Figure S2). To ensure this was a real effect and not due to a solvent/dielectric effect from high concentrations of peptide present, the same experiment was repeated with LtnA2. In this instance, no change in the ${ }^{31} \mathrm{P}-\mathrm{NMR}$ of lipid II occurred (Figure S3). Chemical shift perturbations also occurred in the amide protons of Ala1 and D-Glu2 as well as Lys3-H $\beta$ (Figure S4). The largest ${ }^{1} \mathrm{H}$-chemical shift changes in LtnA1 were observed in the amide N-H signals of His23, Glu24, Cys25, Met26, Trp28, Cys29 and Lys30 (Figure S5). 7 intermolecular NOEs were also found (Figure S6), between D-Glu2 (lipid II) and Asn15, Cys19 and Abu20 (LtnA1), as well as Lys3 (lipid II) and Asn15 (LtnA1). The chemical shift assignments and NOE peak list from the LtnA1:lipid II complex were then used to generate a model of the complex using CYANA (Figure 6 and S7) ${ }^{30}$ Based on the observed ${ }^{31} \mathrm{P}$ chemical shift perturbations and intermolecular NOEs, as well as the earlier work of Sahl et al, ${ }^{13 \mathrm{~d}}$ four loose artificial NOE restraints were introduced involving residues Glu24 and Lys30 with both phosphate atoms for relative anchoring of LtnA1 and lipid II. The resulting CYANA generated model is consistent with all intermolecular NOEs and chemical shift perturbations.

LtnA1 binds to the pyrophosphate and pentapeptide moieties of lipid II (Figure 6A). Closer analysis of the structure revealed the D-ring (res. 22-30) of LtnA1 forms a hydrophilic groove that binds to the pyrophosphate on lipid II (Figure 6B). The LtnA1:lipid II complex was analyzed in MacPyMol to identify intermolecular interactions between LtnA1 and lipid II. The amide protons of Glu24, Cys25, Met26 and Lys30 form hydrogen bonds with the phosphate oxygen atoms in lipid II (Figure 6C). Furthermore, the electron deficient imidazole ring on His 23 forms a pi-anion bond ${ }^{31}$ (piclamp) with a P-O oxygen anion in lipid II, as well as a H-bond. Chemical shift perturbations were found in all of these LtnA1 residues, further supporting the CYANA generated LtnA1:lipid II complex.

\section{Comparison to other lipid II binding lantibiotics}

Having identified that LtnA1 binds to the pyrophosphate of lipid II, we next sought to compare this binding mechanism to other lipid II-binding lantibiotics (Figure 7). The NMR solution structure of the nisin:lipid II complex was previously determined in DMSO. ${ }^{13 \mathrm{~d}}$ This Type-A(I) lantibiotic adopts an elongated structure in which the $\mathrm{N}$-terminal residues bind to lipid II (Figure 7A). In contrast, as a Type-B-like lantibiotic, LtnA1 adopts a globular structure, with lipid II binding occurring at the C-terminus (Figure 7B). The pyrophosphate cage adopted by nisin (Figure 7D) is shallower than that adopted by LtnA1 (Figure 7E), with binding occurring exclusively through amide intermolecular hydrogen bonds. As well as intermolecular hydrogen bonding, LtnA1 also uses an interesting pi-clamp interaction to bind to the lipid II pyrophosphate. For both nisin and LtnA1, the major interaction is with the lipid II pyrophosphate. The NMR solution structure of mersacidin (2) was previously determined in DPC micelles containing Gram-positive lipid II. ${ }^{14 \mathrm{~b}}$ However, the NMR solution structure adopted by lipid II in this complex was not determinable. Mersacidin has significantly higher affinity for lipid II than lipid I (which lacks GlcNAc) $)^{32}$ and has increased activity in calcium enriched media. ${ }^{33}$ These findings suggest that mersacidin may bind to both the pyrophosphate and GlcNAc moieties in lipid II. In the absence of a structure of the mersacidin:lipid II complex, lipid II analogue 9 was
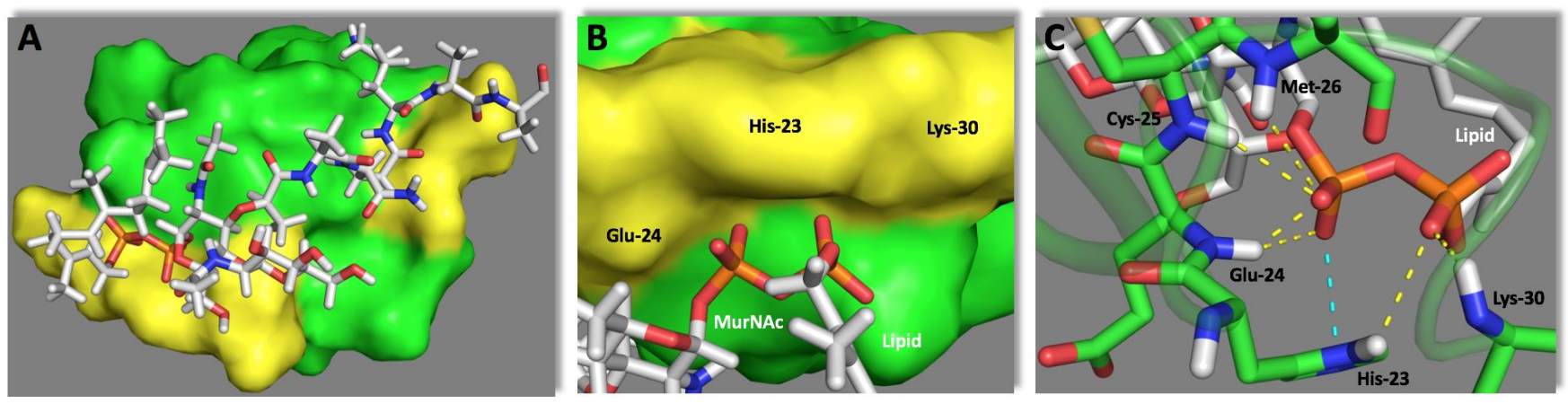

Figure 6. (A) Model of the complex formed between LtnA1 and (E,E)-farnesyl lipid II. LtnA1 is shown as a surface representation with hydrophobic residues green and hydrophilic residues yellow. Lipid II is represented as a stick model. LtnA1 binds mainly to the pyrophosphate and pentapeptide regions of lipid II. (B) The pyrophosphate binding pocket of LtnA1 as a surface representation. (C) Key binding interactions between the C-terminal residues of LtnA1 and pyrophosphate of lipid II. H-bonds between the lipid II pyrophosphate and the amide protons of Glu24, Cys25, Met26 and Lys30 were identified (yellow dash), as well as a possible pianion interaction (turquoise dash) and H-bond between the lipid II pyrophosphate and the imidazole ring of His 23. 

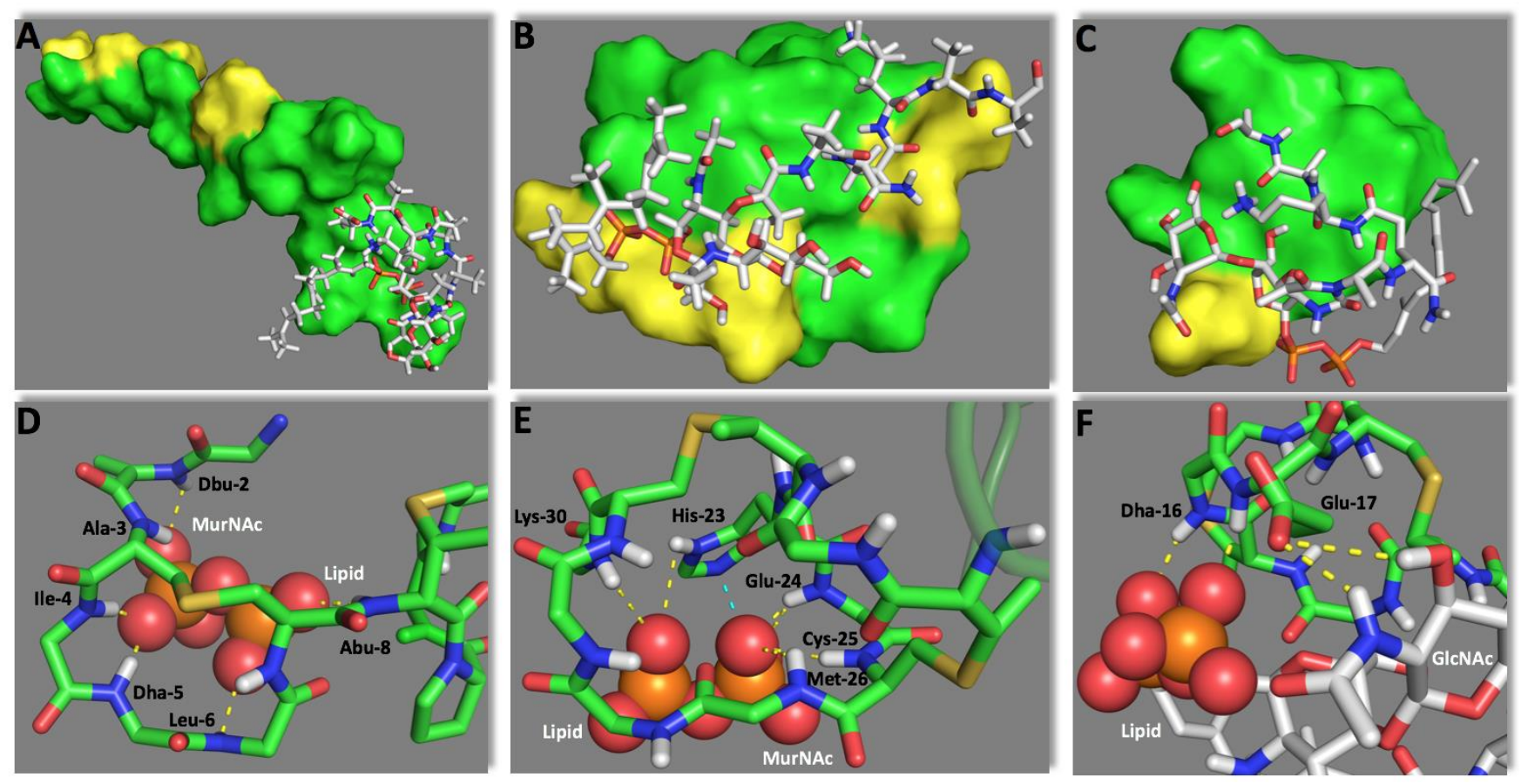

Figure 7. (A) NMR solution structure of the nisin-lipid II complex (1WCO), (B) Model of the LtnA1-lipid II complex and (C) Lipid II docked into the NMR solution structure of mersacidin bound to lipid II in DPC micelles (1MQZ). In (A) - (C) peptides are shown as a surface representation with hydrophobic residues green and hydrophilic residues yellow, and lipid II is represented as a stick model. Nisin adopts an elongated structure and binds to lipid II with its N-terminal residues, whereas both LtnA1 and mersacidin adopt a globular structure and bind to lipid II through their C-terminal residues. (D) The nisin-lipid II pyrophosphate cage, $(\mathbf{E})$ the LtnA1-lipid II cage and (F) a model of the proposed mersacidin-lipid II intermolecular bonds. In (D) - (F) the peptide backbone is shown a stick model wherein carbon $=$ green, oxygen $=$ red, nitrogen $=$ blue and sulfur $=$ yellow, and some side-chain residues have been included. The pyrophosphate moiety within lipid II is represented by spheres with the rest of lipid II omitted for clarity, unless needed to show key intermolecular bonds. Nisin forms a tight pyrophosphate cage with several intermolecular hydrogen bonds, whereas the LtnA1 pyrophosphate cage is looser, with less intermolecular hydrogen-bonds but with a pi-clamping interaction. The mersacidin-lipid II model suggests hydrogen-bonding between Glu-17 and both the pyrophosphate and GlcNAc moieties of lipid II and is supported by all experimental evidence of mersacidin-lipid II binding to date.

docked into the NMR solution structure of mersacidin using AutoDock Vina (Figure $7 \mathrm{C}$ and F). ${ }^{34}$ This Type-B lantibiotic adopts a globular structure similar to LtnA1. In the resulting model, the C-terminal residues of mersacidin that have previously been shown to interact with lipid II are involved in lipid II binding. There are predicted intermolecular $\mathrm{H}$-bonds between the amides of Dha16 and Glu17 and the pyrophosphate, as well as the carboxylate group of Glu17 and GlcNAc. Glu17 was previously shown be very important in the antimicrobial activity of mersacidin, with mutation of this residue to alanine abolishing activity. ${ }^{18}$ Care must be taken when comparing the structures of nisin, LtnA1 and mersacidin bound to lipid II, as they were calculated in different solvents. Ideally these would be calculated in the same solvent system for comparison, however this is not possible due to the differing solubility's of the respective peptide:lipid II complexes.

Haloduracin is another two-component lantibiotic, composed of Type-B-like (Hala, (3)) and Type-A(I)-like (Hal $\beta$ (4)) peptides. ${ }^{16 a}$ Analogues to the lacticin 3147 peptides, Hal $\alpha$ and Hal $\beta$ operate synergistically in a 1:1 ratio to kill Gram-positive bacteria. Previous studies have also shown that Hal $\alpha$ inhibits the transglycosylation reaction between lipid II and penicillin-binding protein $1 \mathrm{~b}$ (PBP1b) by binding to lipid II in a 2:1 ratio. ${ }^{35}$ These findings suggest a 2:2:1 Hal $\alpha: H a l \beta: l i p i d$ II complex is formed, which inhibits cell wall biosynthesis and mediates pore formation. Previous studies on the nisin:lipid II binding interaction by ITC showed a 1:1 binding ratio, however further in vivo studies using fluorescently labelled lipid II suggested a 2:1 nisin:lipid II ratio in the resulting complex formed. ${ }^{36}$ Like Hal $\alpha$ and nisin, our studies suggest that LtnA1 binds to lipid II in a 2:1 ratio. Furthermore, given the 1:1 synergistic activity of LtnA1 and LtnA2, our results suggest that lacticin 3147 exerts its antimicrobial effect by a similar mechanism to haloduracin - by forming a 2:2:1 LtnA1:LtnA2:lipid II complex, that results in rapid membrane lysis by pore formation.

\section{CONCLUSIONS}

In this article we have shown that the lantibiotic lacticin 3147 can kill Gram-positive bacteria by several mechanisms of action. The type-B-like component of lacticin 3147 , LtnA1, is bacteriostatic and does not form pores in the bacterial membrane. Instead, LtnA1 binds 
to the peptidoglycan precursor lipid II in a 2:1 ratio by formation of a "pyrophosphate cage" using its Cterminal residues, which likely inhibits peptidoglycan biosynthesis. The other component, LtnA2, is bactericidal at micromolar concentrations, killing bacteria by membrane lysis. Co-administration of both LtnA1 and LtnA2 causes rapid lysis in artificial membranes without lipid II, indicating direct pore forming activity by this peptide combination. However, the presence of lipid II greatly enhances this activity. These results provide valuable insight into the mechanism of action of lipid II-binding lantibiotics, as now at least three (nisin, haloduracin and lacticin 3147) are known to bind to lipid II in a 2:1 ratio. Analogous to the mechanism of action of haloduracin, lacticin 3147 appears to form a 2:2:1 peptide A:peptide B:lipid II complex, that results in rapid membrane lysis by pore formation. LtnA1 and Hal $\alpha$ both bind to lipid II on the surface of the cell membrane and then direct their partner peptide to the membrane to kill bacteria. This may allow for the delivery of different antimicrobial compounds across the cell membrane, as has been shown for other antimicrobial peptides. ${ }^{37}$ Although we now have a detailed understanding of how lacticin 3147 interacts with lipid II, the synergistic interaction between LtnA1 and LtnA2 at the molecular level is less well understood. Further studies are ongoing in this area.

\section{ASSOCIATED CONTENT}

\section{Supporting Information}

Additional ITC data, pore-formation assay results, timekill assays, SYTOX green assays, NMR data, CYANA data, and experimental procedures. This material is available free of charge via the internet at http://pubs.acs.org.

\section{AUTHOR INFORMATION}

\section{Corresponding Author}

*john.vederas@ualberta.ca

\section{ORCID}

Stephen A. Cochrane: 0000-0002-6239-6915; Ryan T. McKay: 0000-0002-0255-159X; John C. Vederas: 00000002-2996-0326; Alireza Bakhtiary: 0000-0001-91026932.

\section{Funding Sources}

We thank The Natural Sciences and Engineering Research Council of Canada, Alberta Innovates Health Solutions and the Wellcome Trust (110270/A/15/Z) for funding.

\section{Notes}

The authors declare no competing financial interest

\section{ACKNOWLEDGMENTS}

We thank Wayne Moffat, Brandon Findlay and Gareth Lambkin for assistance with NMR experiments, fluorescence experiments, biochemical assays respectively. Alberta
Innovates Health Solutions and the Natural Sciences \& Engineering Research Council of Canada provided funding.

\section{REFERENCES}

(1) World Health Organization. Antimicrobial resistance: 2014 global report on surveillance.; 2014.

(2) (a) Ling, L. L.; Schneider, T.; Peoples, A. J.; Spoering, A. L.; Engels, I.; Conlon, B. P.; Mueller, A.; Schäberle, T. F.; Hughes, D. E.; Epstein, S.; Jones, M.; Lazarides, L.; Steadman, V. A.; Cohen, D. R.; Felix, C. R.; Fetterman, K. A.; Millett, W. P.; Nitti, A. G.; Zullo, A. M.; Chen, C.; Lewis, K. Nature 2015, 517, 455-459; (b) Zipperer, A.; Konnerth, M. C.; Laux, C.; Berscheid, A.; Janek, D.; Weidenmaier, C.; Burian, M.; Schilling, N. A.; Slavetinsky, C.; Marschal, M.; Willmann, M.; Kalbacher, H.; Schittek, B.; Brötz-Oesterhelt, H.; Grond, S.; Peschel, A.; Krismer, B. Nature 2016, 535, 511-516; (c) Cociancich, S.; Pesic, A.; Petras, D.; Uhlmann, S.; Kretz, J.; Schubert, V.; Vieweg, L.; Duplan, S.; Marguerettaz, M.; Noëll, J.; Pieretti, I.; Hügelland, M.; Kemper, S.; Mainz, A.; Rott, P.; Royer, M.; Süssmuth, R. D. Nat Chem Biol 2015, 11, 195-197; (d) Chu, J.; Vila-Farres, X.; Inoyama, D.; Ternei, M.; Cohen, L. J.; Gordon, E. A.; Reddy, B. V.; Charlop-Powers, Z.; Zebroski, H. A.; Gallardo-Macias, R.; Jaskowski, M.; Satish, S.; Park, S.; Perlin, D. S.; Freundlich, J. S.; Brady, S. F. Nat Chem Biol 2016, 12, 1004-1006.

(3) Alvarez-Sieiro, P.; Montalbán-López, M.; Mu, D.; Kuipers, O. P. Appl Microbiol Biotechnol 2016, 100, 2939-2951.

(4) Tang, W.; Jiménez-Osés, G.; Houk, K. N.; van der Donk, W. A. Nat Chem 2015, 7, 57-64; (b) Arnison, P.G. et al. Nat. Prod. Rep. 2013, 30, 108-160; (c) Knerr, P.J.; van der Donk, W.A. Annu. Rev. Biochem. 2012, 81, 479-505.

(5) Koopmans, T.; Wood, T. M.; t Hart, P.; Kleijn, L. H.; Hendrickx, A. P.; Willems, R. J.; Breukink, E.; Martin, N. I. J Am Chem Soc 2015, 137, 9382-9389; (b) Field, D.; Cotter, P.D.; Hill, C.; Ross, R.P. Frontiers Microbiol. 2015, 6, doi: 10.3389/fmicb.2015.01363.

(6) (a) Cruz, J. C.; Iorio, M.; Monciardini, P.; Simone, M.; Brunati, C.; Gaspari, E.; Maffioli, S. I.; Wellington, E.; Sosio, M.; Donadio, S. J Nat Prod 2015, 78, 2642-2647; (b) Escano, J.; Smith, L. Expert Opin Drug Discov 2015, 10, 857-870; (c) Tabor, A. B. Bioorg. Chem. 2014, 55, 39-50; (d) Escano, J.; Smith, L. Exp. Opin. Drug Discovery 2015, 10, 857-870.

(7) (a) Oppedijk, S. F.; Martin, N. I.; Breukink, E. Biochim. Biophys. Acta 2016, 1858, 947-957; (b) Münch, D.; Sahl, H. G. Biochim. Biophys. Acta 2015, 1848, 3062-3071; (c) Bush, K. Rev Sci Tech 2012, 31, 43-56.

(8) Perkins, H. R. Biochemical Journal 1969, 111, 195-205.

(9) Schneider, T.; Kruse, T.; Wimmer, R.; Wiedemann, I.; Sass, V.; Pag, U.; Jansen, A.; Nielsen, A. K.; Mygind, P. H.; Raventós, D. S.; Neve, S.; Ravn, B.; Bonvin, A. M.; De Maria, L.; Andersen, A. S.; Gammelgaard, L. K.; Sahl, H. G.; Kristensen, H. H. Science 2010, 328, 1168-1172.

(10) Cochrane, S. A.; Findlay, B.; Bakhtiary, A.; Acedo, J. Z.; Rodriguez-Lopez, E. M.; Mercier, P.; Vederas, J. C. Proc Natl Acad Sci U S A 2016, 113, 11561-11566.

(11) Martin, N. I.; Breukink, E. Future Microbiol 2007, 2, 513-525.

(12) McAuliffe, O.; Ross, R. P.; Hill, C. FEMS Microbiol Rev 2001, 25, 285-308.

(13) (a) Breukink, E.; Wiedemann, I.; van Kraaij, C.; Kuipers, O. P.; Sahl, H. G.; de Kruijff, B. Science 1999, 286, 2361-2364; (b) Wiedemann, I.; Breukink, E.; van Kraaij, C.; Kuipers, O. P.; Bierbaum, G.; de Kruijff, B.; Sahl, H. G. J Biol Chem 2001, 276, 1772-1779; (c) Breukink, E.; van Heusden, H. E.; Vollmerhaus, P. J.; Swiezewska, E.; Brunner, L.; Walker, S.; Heck, A. J.; de Kruijff, B. J Biol Chem 2003, 278, 19898-19903; (d) Hsu, S. T.; Breukink, E.; Tischenko, E.; Lutters, M. A.; de Kruijff, B.; Kaptein, R.; Bonvin, A. M.; van Nuland, N. A. Nat Struct Mol Biol 2004, 11, 963-967; (e) Hart, P.; Oppedijk, S. F.; Breukink, E.; Martin, N. I. Biochemistry 2016, 55, 232-237.

(14) (a) Brötz, H.; Bierbaum, G.; Leopold, K.; Reynolds, P. E.; Sahl, H. G. Antimicrob Agents Chemother 1998, 42, 154-160; 
(b) Hsu, S. T.; Breukink, E.; Bierbaum, G.; Sahl, H. G.; de Kruijff, B.; Kaptein, R.; van Nuland, N. A.; Bonvin, A. M. J Biol Chem 2003, 278, 13110-13117; (c) Sass, P.; Jansen, A.; Szekat, C.; Sass, V.; Sahl, H. G.; Bierbaum, G. BMC Microbiol 2008, 8, 186; (d) Böttiger, T.; Schneider, T.; Martínez, B.; Sahl, H. G.; Wiedemann, I. Appl Environ Microbiol 2009, 75, 4427-4434.

(15) (a) Islam, M. R.; Nishie, M.; Nagao, J.; Zendo, T.; Keller, S.; Nakayama, J.; Kohda, D.; Sahl, H. G.; Sonomoto, K. J Am Chem Soc 2012, 134, 3687-3690. (b) Elsayed, K. M.; Islam, M. R.; Al-Mahin, A.; Nagoa, J.; Zendo, T.; Sonomoto, K. J. Biosci. Engineer. 2017, 3, 398-401.

(16) (a) McClerren, A. L.; Cooper, L. E.; Quan, C.; Thomas, P. M.; Kelleher, N. L.; van der Donk, W. A. Proc Natl Acad Sci U S A 2006, 103, 17243-17248. (b) Lawton, E. M.; Cotter, P. D.; Hill, C.; Ross, R. P. FEMS Microbiol Lett 2007, 267, 64-71. (c) Cooper, L. E.; McClerren, A. L.; Chary, A.; van der Donk, W. A. Chem Biol 2008, 15, 1035-1045.

(17) (a) Ryan, M. P.; Rea, M. C.; Hill, C.; Ross, R. P. Appl Environ Microbiol 1996, 62, 612-619. (b) Ryan, M. P.; Jack, R. W.; Josten, M.; Sahl, H. G.; Jung, G.; Ross, R. P.; Hill, C. J Biol Chem 1999, 274, 37544-37550. (c) Martin, N. I.; Sprules, T.; Carpenter, M. R.; Cotter, P. D.; Hill, C.; Ross, R. P.; Vederas, J. C. Biochemistry 2004, 43, 3049-3056. (d) Morgan, S. M.; O'connor, P. M.; Cotter, P. D.; Ross, R. P.; Hill, C. Antimicrob Agents Chemother 2005, 49, 2606-2611.

(18) Cotter, P. D.; Deegan, L. H.; Lawton, E. M.; Draper, L. A.; O’Connor, P. M.; Hill, C.; Ross, R. P. Mol Microbiol 2006, 62, 735-747.

(19) Oman, T. J.; van der Donk, W. A. ACS Chem Biol 2009, 4, 865-874.

(20) Wiedemann, I.; Böttiger, T.; Bonelli, R. R.; Wiese, A.; Hagge, S. O.; Gutsmann, T.; Seydel, U.; Deegan, L.; Hill, C.; Ross, P.; Sahl, H. G. Mol Microbiol 2006, 61, 285-296.

(21) Pattabiraman, V. R.; McKinnie, S. M.; Vederas, J. C. Angew Chem Int Ed 2008, 47, 9472-9475.

(22) Liu, H.; Pattabiraman, V. R.; Vederas, J. C. Org Lett 2009, 11, 5574-5577.
(23) Liu, W.; Chan, A. S.; Liu, H.; Cochrane, S. A.; Vederas, J. C. J Am Chem Soc 2011, 133, 14216-14219.

(24) Islam, M. R.; Nishie, M.; Nagao, J.; Zendo, T.; Keller, S.; Nakayama, J.; Kohda, D.; Sahl, H. G.; Sonomoto, K. J Am Chem Soc 2012, 134, 3687-3690.

(25) t Hart, P.; Oppedijk, S. F.; Breukink, E.; Martin, N. I. Biochemistry 2016, 55, 232-237.

(26) Malvern Instruments Limited, The Working Principle of Isothermal Titration Calorimetry, July $9^{\text {th }}, 2015$.

(27) Mohammadi, T.; van Dam, V.; Sijbrandi, R.; Vernet, T.; Zapun, A.; Bouhss, A.; Diepeveen-de Bruin, M.; Nguyen-Distèche, M.; de Kruijff, B.; Breukink, E. EMBO J 2011, 30, 1425-1432.

(28) Molenaar, D.; Abee, T.; Konings, W. N. Biochim Biophys Acta 1991, 1115, 75-83.

(29) Langsrud, S.; Sundheim, G. J Appl Bacteriol 1996, 81, 411-418.

(30) Güntert, P.; Mumenthaler, C.; Wüthrich, K. J Mol Biol 1997, 273, 283-298.

(31) Schottel, B. L.; Chifotides, H. T.; Dunbar, K. R. Chem Soc Rev 2008, 37, 68-83.

(32) Brötz, H.; Bierbaum, G.; Reynolds, P. E.; Sahl, H. G. Eur J Biochem 1997, 246, 193-199.

(33) Barrett, M. S.; Wenzel, R. P.; Jones, R. N. Diagn Microbiol Infect Dis 1992, 15, 641-644.

(34) Trott, O.; Olson, A. J. J Comput Chem 2010, 31, 455461.

(35) Oman, T. J.; Lupoli, T. J.; Wang, T. S.; Kahne, D.; Walker, S.; van der Donk, W. A. J Am Chem Soc 2011, 133, 17544-17547.

(36) Hasper, H. E.; de Kruijff, B.; Breukink, E. Biochemistry 2004, 43, 11567-11575.

(37) (a) Vaara, M.; Vaara, T. Nature 1983, 303, 526-528. (b) Cochrane, S. A.; Vederas, J. C. Int J Antimicrob Agents 2014, 44, 493-499. (c) Cochrane, S. A.; Li, X.; He, S.; Yu, M.; Wu, M.; Vederas, J. C. J Med Chem 2015, 58, 9779-9785. 